\title{
Absolute beam emittance measurements at RHIC using ionization profile monitors
}

\author{
M. Minty, R. Connolly, C. Liu, \\ T. Summers, S. Tepikian
}

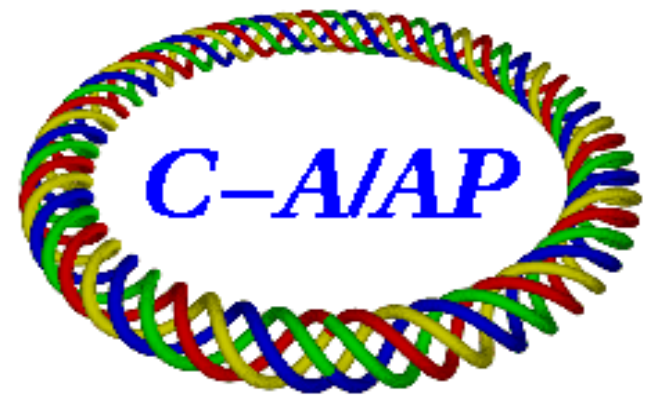

\section{Collider-Accelerator Department Brookhaven National Laboratory Upton, NY 11973}

Notice: This document has been authorized by employees of Brookhaven Science Associates, LLC under Contract No. DE-AC02-98CH10886 with the U.S. Department of Energy. The United States Government retains a nonexclusive, paid-up, irrevocable, world-wide license to publish or reproduce the published form of this document, or allow others to do so, for United States Government purposes. 


\title{
ABSOLUTE BEAM EMITTANCE MEASUREMENTS AT RHIC USING IONIZATION PROFILE MONITORS
}

\author{
M. Minty", R. Connolly, C. Liu, T. Summers, S. Tepikian \\ Brookhaven National Laboratory, Upton, NY 11973, U.S.A.
}

\section{INTRODUCTION}

In the past, comparisons between emittance measurements [1] obtained using ionization profile monitors, Vernier scans (using as input the measured rates from the zero degree counters, or ZDCs [2]), the polarimeters and the Schottky detectors evidenced significant variations of up to 100\% [1]. In this report we present studies of the RHIC ionization profile monitors (IPMs). After identifying and correcting for two systematic instrumental errors in the beam size measurements, we present experimental results showing that the remaining dominant error in beam emittance measurements at RHIC using the IPMs was imprecise knowledge of the local beta functions. After removal of the systematic errors and implementation of measured [3] beta functions, precise emittance measurements result. Also, consistency between the emittances measured by the IPMs and those derived from the ZDCs was demonstrated.

\section{BRIEF HISTORY OF THE RHIC IPMS}

The design of the RHIC IPMs has evolved over time with continuous improvements. The first prototype was built and tested in 1996 [4] with first measurements in RHIC in 1999 [5, 6]. In 2002 two changes were made motivated by experiences with beam: shielding was added upstream of the detectors to prevent signal contributions from upstream beam losses and the electrodes were made longer to avoid electron clouds from migrating into the region of the detector [7]. In 2005 fast signal gating was added to avoid depletion of the multichannel plate (MCP) detector and better isolation of the detector from the electromagnetic fields of the beam was implemented [7]. As the beam intensities increased, this latter effect was further suppressed with a new design in 2007 which placed all electronics inside a Faraday cage outside of the path of the beam's image current [8]. The prototype for this new design [8] was implemented for the Yellow Ring vertical plane (YV) in 2008. In 2010 the new design [8] was implemented in both the Blue and Yellow Ring horizontal detectors (BH, YH) and these IPMs were moved to locations where the beam sizes were larger. The new IPM design [8] was implemented in the last remaining plane, Blue Ring vertical (BV), in 2013.

\section{INSTRUMENTAL SYSTEMATIC ERRORS}

During the FY11 RHIC run [9], it was found that when the beams were brought into collision at a new third experiment (AnDY), the vertical beam size measurements from the IPMs changed considerably. To investigate further measurements were taken while scanning the beam across the area of the detector. The measurements revealed significant damage to the multi-channel plate detectors (MCP) due to depletion [10] however with corrections applied, the position sensitivity still remained (since the beam sizes at full energy were small compared to the region of the MCP depletion). Other issues concerned variations between measurements, which were not small compared to expectation based on the statistical properties (i.e. ionization cross sections) of the measurements, and channel-to-channel variations within a single measurement (see for example measurements in Ref. [8]).

\section{CHANNEL-BY-CHANNEL OFFSET CORRECTION AND GAIN CALIBRATIONS}

A conceptual view of the IPM and photographs are shown in Fig. 1. The signals from the MCP (bottom left) are processed through 64 channels as seen on the anode board (bottom right) and transferred to amplifiers through 64 ceramic-beaded wires (bottom left). Using the previously acquired data (beam size measurements as a function of beam centroid position), the channel offsets were determined by measurements with beam passing across the MCPs but not above the specific channels of interest. After applying the offset corrections to all the measured profiles and removing bad channels, the gain calibrations were obtained using the following automated procedure. For each IPM:

(1) Fit each of the (20 to 30 or so) profiles in the calibration scan with a Gaussian and compute the chi-squared, $\chi^{2}$.

(2) Calculate a figure of merit equal to the mean chi-squared $\left\langle\chi^{2}\right\rangle$.

(3) For a given channel (the RHIC IPMs have 64 readout channels), scale the channel gain and repeat steps 1-2.

(4) Iterate steps 1-3 over a range of channel gain scale factors.

(5) Perform a polynomial fit to the resultant $\left\langle\chi^{2}\right\rangle$ versus scaled channel gain. Examples are shown in Fig. 2.

(6) Implement the channel gain so found (with minimum $<\chi^{2}>$ ).

(7) Repeat steps 1-6 for all 64 channels. 


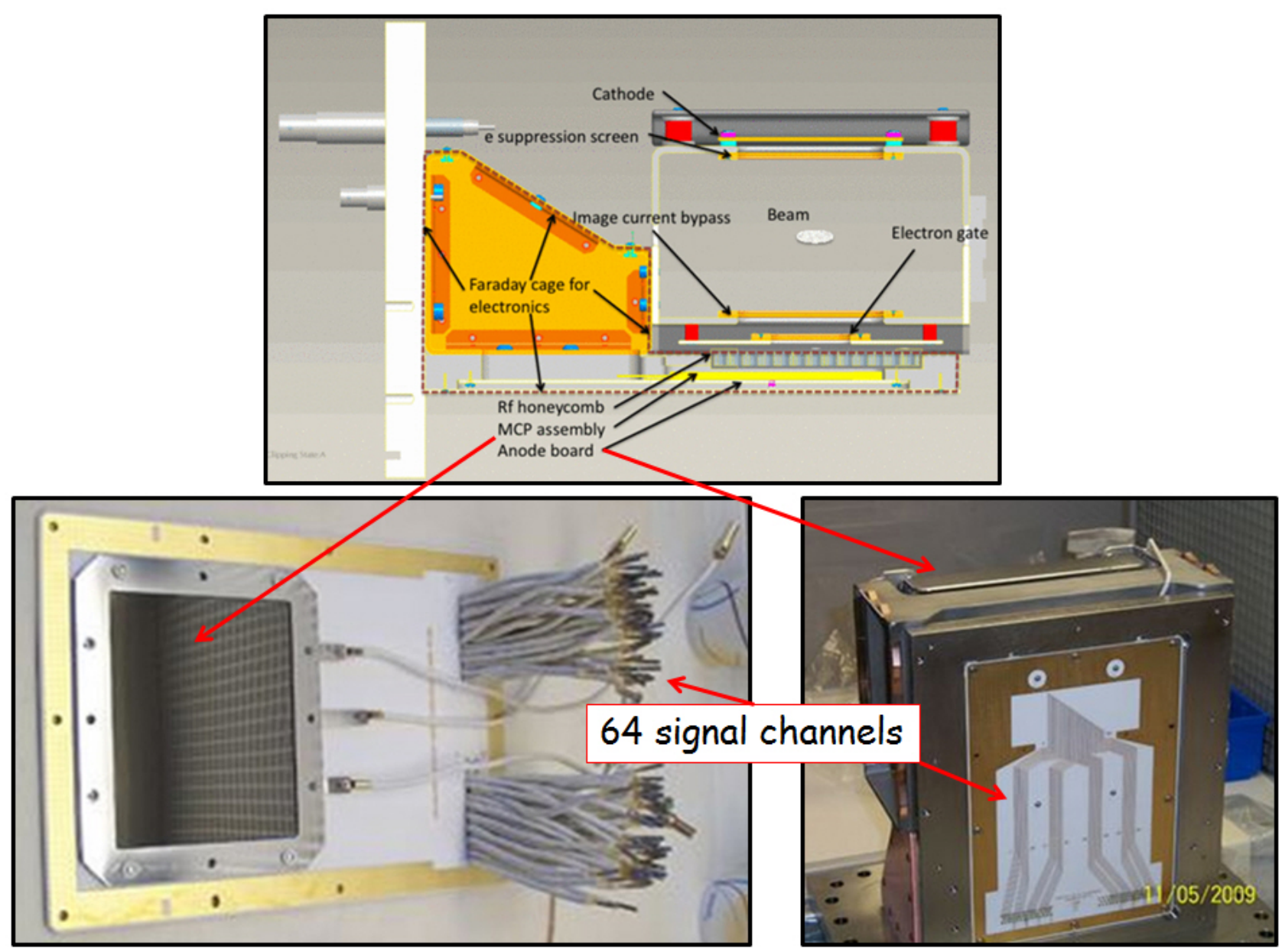

Figure 1: Conceptual view (top) and photographs (bottom) of new RHIC IPM [8]. The multichannel plate (MCP, left) dimensions are $8 \mathrm{~cm}$ by $10 \mathrm{~cm}$. The signals from the MCP are collected by the 64-channel anode board (right) and read out through the ceramic-beaded wires (left).

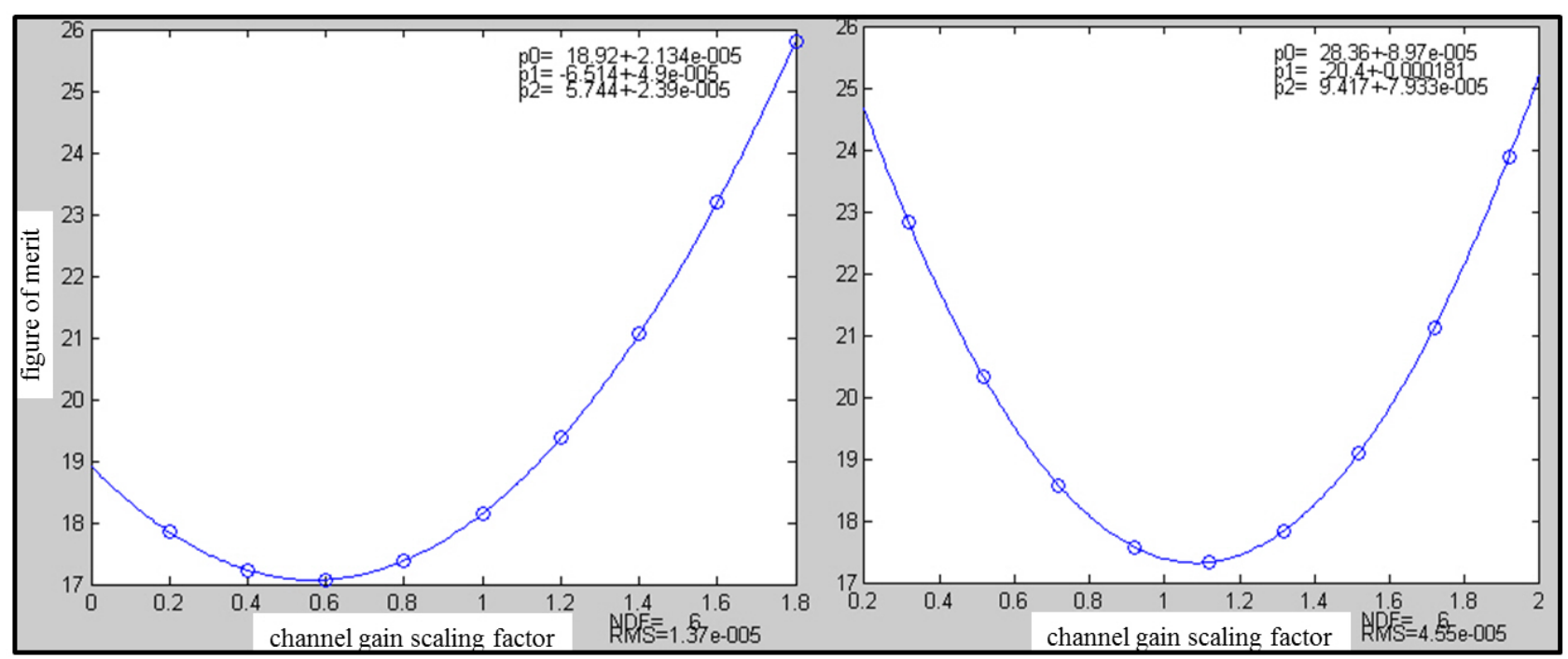

Figure 2: Examples of polynomial fit to figure of merit, $\left\langle\chi^{2}\right\rangle$, versus channel gain scaling factor for two different IPM channels. 
Figure 3 shows IPM profile measurements from the FY11 RHIC run [8] without channel-by-channel corrections (left) and from the FY12 RHIC run with corrections (right). The new and simplified header display now includes a fit error which until these corrections were applied was not useful in evaluation of the quality of the profile measurements.
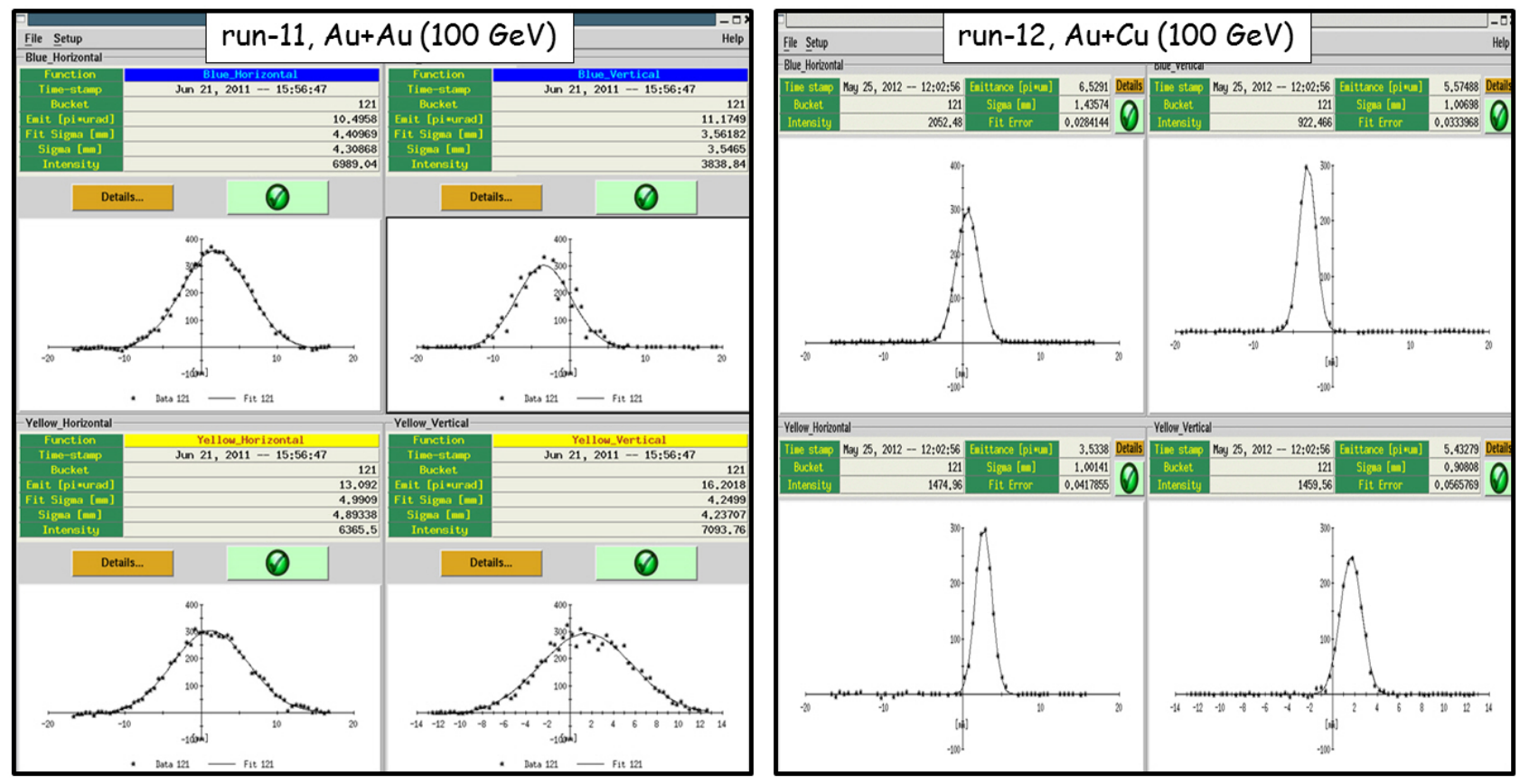

Figure 3: Example beam profile measurements before (left) and after (right) implementation of IPM offset and gain corrections. Shown in the top row are the Blue ring horizontal (left) and vertical (right) profiles and in the bottom row the Yellow ring horizontal (left) and vertical (right) profiles.

The Gaussian fit algorithm was considerably better constrained after implementing the channel-by-channel offset corrections and gain calibrations. For example, shown in Fig. 4 are the vertical rms beam sizes as obtained from Gaussian fits (ref. Fig. 3) to the beam profiles measured using the IPMs in the Blue (left) and Yellow (right) Rings. The step changes correspond to the time at which the vertical separation bumps, which span the IPMs, were removed to bring the beams in collision at a third colliding beam experiment. A subset of the raw profiles shown in the figure were reanalyzed after applying the channel-by-channel offset corrections and gain calibrations. The results are summarized in Tables 1 (Blue Ring) and 2 (Yellow Ring). We conclude from this study that the observed changes in vertical beam size, introduced when the beams were brought into collision at the third interaction point, were artificial.
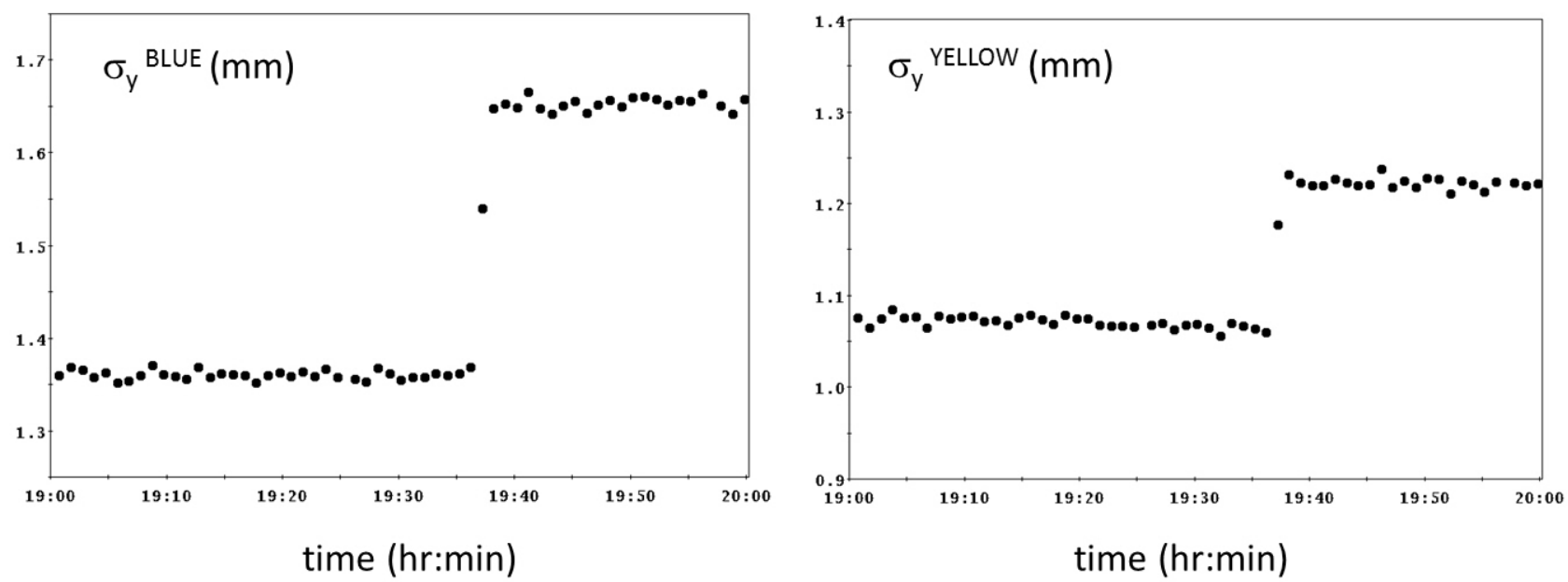

Figure 4: Vertical beam sizes $\sigma_{\mathrm{y}}$ (in $\mathrm{mm}$ ) versus time measured in the Blue Ring (left) and Yellow Ring (right) evidencing a step change (determined by the analysis presented here to be artificial) when collisions were established at a third interaction point during the FY11 RHIC Run. 


\begin{tabular}{|c|c|c|}
\hline $\begin{array}{c}\text { Number of Interaction } \\
\text { Points }\end{array}$ & $\begin{array}{c}\sigma_{\mathrm{y}}(\mathrm{mm}) \text { without } \\
\text { corrections }\end{array}$ & $\sigma_{\mathrm{y}}(\mathrm{mm})$ with corrections \\
\hline 2 & 1.37 & 1.51 \\
\hline 3 & 1.66 & 1.49 \\
\hline ratio & 1.21 & 0.99 \\
\hline
\end{tabular}

Table 1: Blue Ring - comparison of the vertical beam sizes $\sigma_{\mathrm{y}}$ measured by the RHIC IPMs during nominal operations with 2 interaction points (at PHENIX and STAR) and with 3 interaction points (PHENIX, STAR and AnDY) as reported online (without corrections) and as evaluated offline after application of the channel offset corrections and gain calibrations.

\begin{tabular}{|c|c|c|}
\hline $\begin{array}{c}\text { Number of Interaction } \\
\text { Points }\end{array}$ & $\begin{array}{c}\sigma_{\mathrm{y}}(\mathrm{mm}) \text { without } \\
\text { corrections }\end{array}$ & $\sigma_{\mathrm{y}}(\mathrm{mm})$ with corrections \\
\hline 2 & 1.06 & 1.11 \\
\hline 3 & 1.24 & 1.13 \\
\hline ratio & 1.17 & 1.02 \\
\hline
\end{tabular}

Table 2: Yellow Ring - comparison of the vertical beam sizes $\sigma_{\mathrm{y}}$ measured by the RHIC IPMs during nominal operations with 2 interaction points (at PHENIX and STAR) and with 3 interaction points (PHENIX, STAR and AnDY) as reported online (without corrections) and as evaluated offline after application of the channel offset corrections and gain calibrations.

\section{EMITTANCES MEASUREMENTS USING MEASURED BETA FUNCTIONS}

After removal of the systematic measurement errors in the measured beam sizes, the presence of another significant systematic error became apparent (e.g. unphysical shrinking emittances without accompanying beam loss during acceleration in FY12 and FY13 and during the "rotator ramp" with a special E-Lens optic in FY13). Since the emittance $\varepsilon$ is given by the ratio of the square of the rms beam size $\sigma_{\beta}$ to the beta function $\beta$ at the location of the IPM, the value assumed for the beta function was suspect (see the Appendix A for detail on how the emittance is derived from the RHIC IPMs). As an illustration, the reported horizontal and vertical emittances of both the Blue and Yellow beams did not converge to equal values which with stochastic cooling [11] is expected to result [11,12]. This was the case in the FY11 RHIC Run when stochastic cooling was applied in the vertical planes with damping of both transverse planes achieved through betatron coupling [11]. Similarly, in FY14 with full 3-D stochastic cooling [12], the emittances did not converge to identical values as shown in Fig. 5 (upper plot).

During the FY13 and FY14 RHIC Runs, the beta functions measured at the beam position monitors bracketing the IPMs were used to interpolate to the positions of the IPMs [3]. A comparison between the model beta functions (used to date for derivation of the emittances from the beam profile measurements) and the measured beta functions obtained early in the FY14 Run ${ }^{1}$ is given in Table 3. The measured beta functions were used ${ }^{2}$ to correct the measurements (by scaling by the ratio $\beta_{\text {model }} / \beta_{\text {meas }}$ ). Shown in Fig. 5 (bottom) are the emittances obtained using the measured beta functions rather than the model beta functions (which were used in Fig. 5 (top)). The two physics stores shown here ${ }^{3}$ correspond to a time late in the FY14 Run at which time RHIC operations was fully optimized with only occasional adjustments over the course of a physics store to stochastic cooling. The adjustments were made by Operations based on observed relative increased emittances, which was indicative of over-cooling of one beam relative to the other (examples of this are visible in Fig. 5 at $\sim 01: 30$ and 10:00). The convergence of the measurements in all 4 planes to a common value gives good confidence that the emittances are now accurately determined.

\begin{tabular}{|l|c|c|c|c|}
\hline & Blue Horizontal & Blue Vertical & Yellow Horizontal & Yellow Vertical \\
\hline$\beta_{\text {model }}(\mathrm{m})$ & 202 & 118 & 206 & 112 \\
\hline$\beta_{\text {meas }}(\mathrm{m})$ & 262 & 109 & 245 & 174 \\
\hline$\beta_{\text {model }} / \beta_{\text {meas }}$ & 0.77 & 1.08 & 0.84 & 0.64 \\
\hline
\end{tabular}

Table 3: Model and measured beta functions at store energy during the FY14 Au+Au RHIC Run.

\footnotetext{
${ }^{1}$ Blue Ring: tbt.Wed_Mar_26_14:48:13_2014.sdds, Yellow Ring: tbt.Wed_Mar_26_14:48:26_2014.sdds, fill 18124.

${ }^{2}$ See http://www.cadops.bnl.gov/cgi-bin/elog/viewMain.pl?elog=rhic au_2014\&shiftlog=Sun_Mar_30_2014_9:34:50_AM\#20140230145643

${ }^{3}$ Data from 06/07/14-06/08/14, fills 18402-18403.
} 


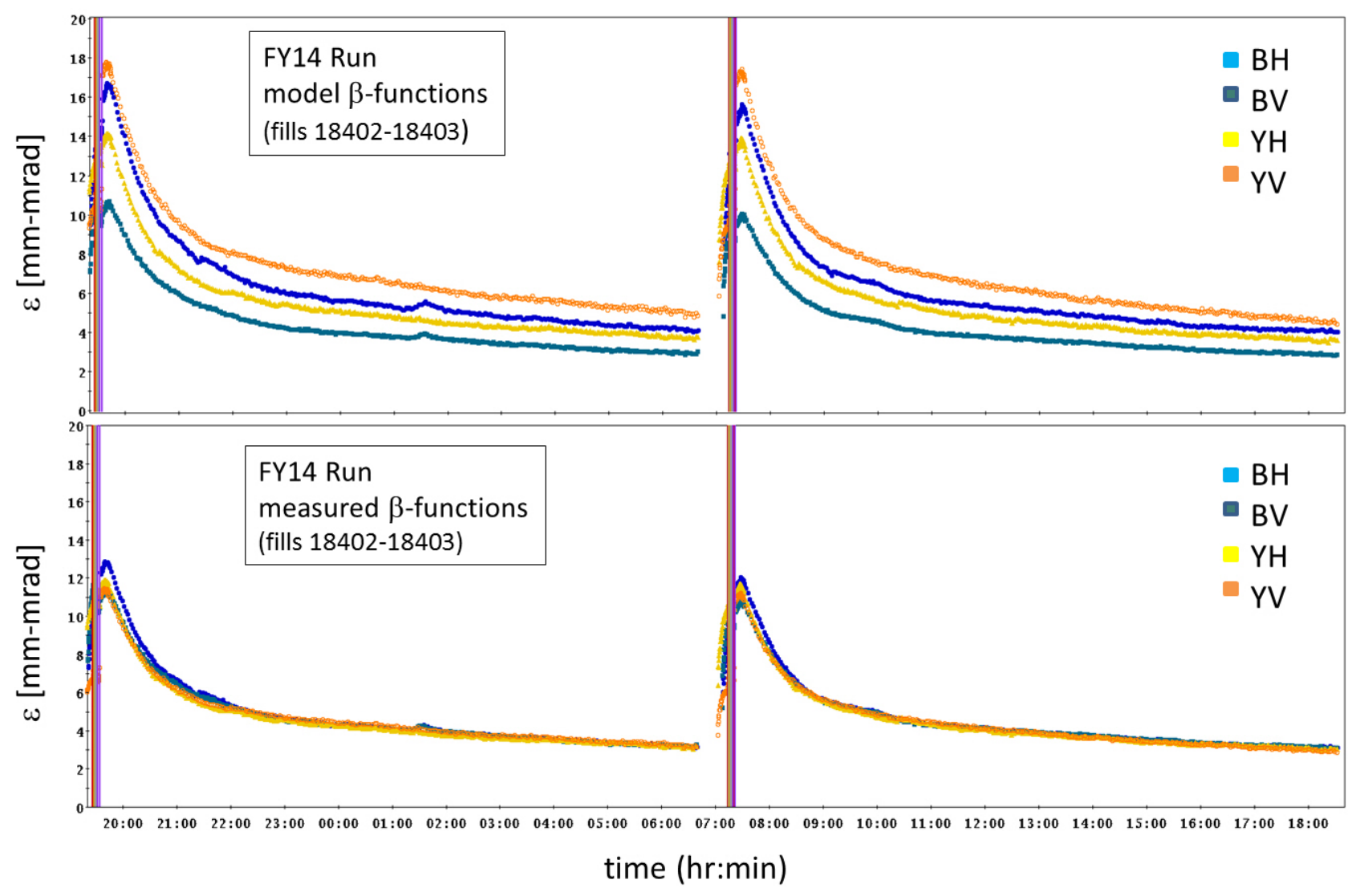

Figure 5: Evolution of the transverse beam emittances with 3D stochastic cooling [12] during the FY14 RHIC Run derived from the IPM profile measurements in all 4 planes (horizontal and vertical in the Blue and Yellow Rings) during two physics stores derived using model beta functions (top) and, for the same two stores, using beta functions interpolated from measurements from nearby beam position monitors (bottom).

\section{COMPARISON OF EMITTANCE MEASUREMENTS FROM THE RHIC IPMS AND FROM THE RHIC ZDCS}

As mentioned in the introduction, emittance comparisons using different methods have in the past shown disparities. Here we compare the emittance measurements from the IPMs to those inferred using the experiment's ZDC counters [2] as input and analyzed in the application StoreAnalysis [13], which among other things computes the effective beam emittance taking into account the measured ZDC rates, the model beta functions (assumed equal in all 4 planes), and the bunch length (for evaluation of the hourglass effect). Shown in Fig. 6 are the rms emittance measurements from the RHIC IPMs and from the ZDCs. In Fig. 6a (FY11 Run $\left.{ }^{4}-\mathrm{Au}+\mathrm{Au}, 100 \mathrm{GeV}\right)$, the IPM measurements did not at that time have channel-by-channel offset corrections or gain calibrations. In Fig. 6b (FY14 Run $\left.{ }^{5}-\mathrm{Au}+\mathrm{Au}, 100 \mathrm{GeV}\right)$ the channel-by-channel offset corrections were applied (with unity gain corrections as these were found not to be necessary since removal of certain unused electronics during the preceding shutdown [14]). Figure 6c shows the same data as Fig. $6 \mathrm{~b}$ now with the measured beta functions applied at the IPMs. Figure $6 \mathrm{~d}$ shows the same data as Fig. $6 \mathrm{~b}$ using measured beta functions at the IPMs and at the ZDCs (see Appendix B for derivations). From these results we observe that with all corrections applied the agreement between emittance measurements from the RHIC IPMs and the ZDC counters is considerably improved.

\footnotetext{
${ }^{4}$ Data from 06/20/11, fill 16091.

${ }^{5}$ Data from 05/04/14, fill 18277.
} 


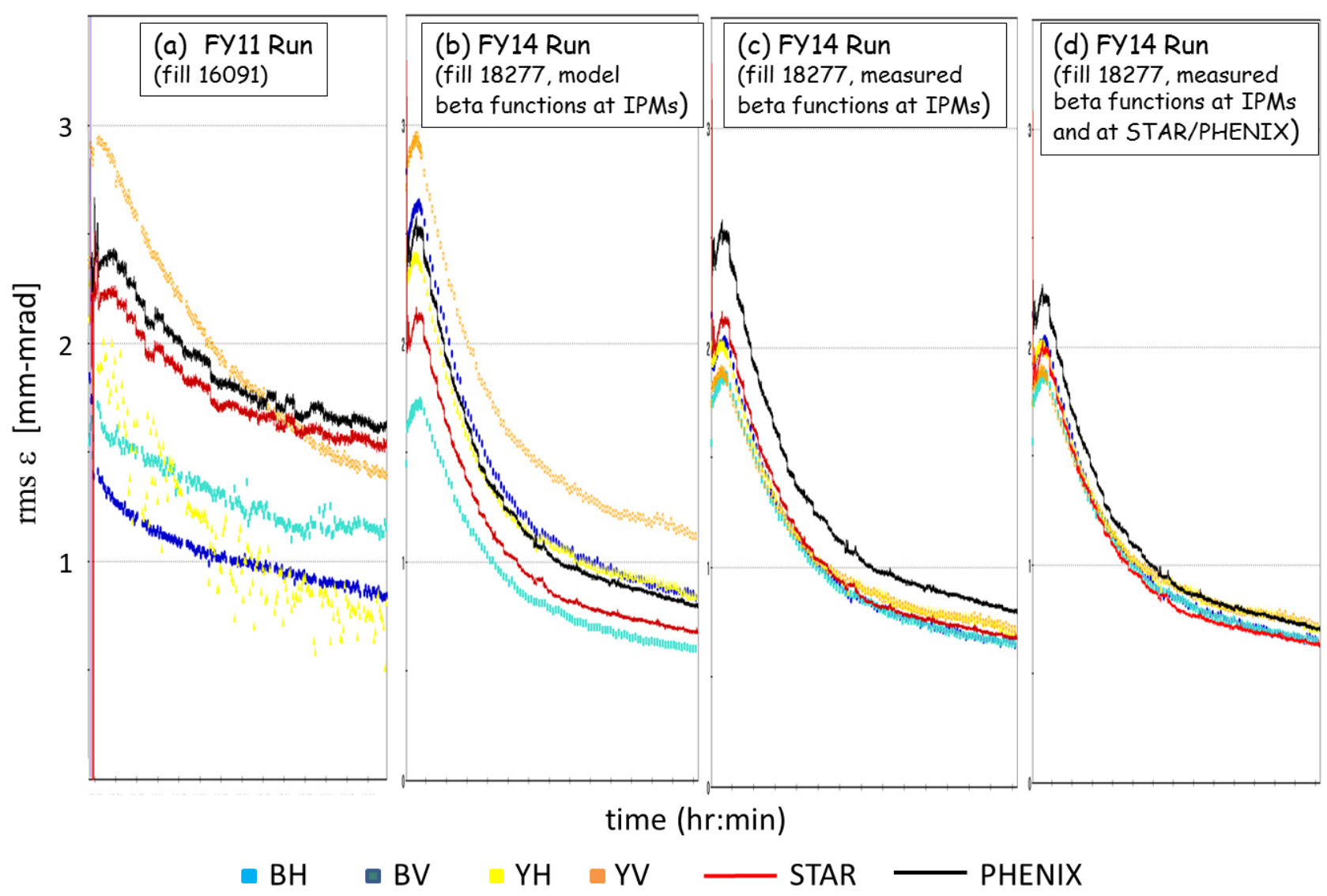

Figure 6: Comparison of rms emittance measurements during $\mathrm{Au}+\mathrm{Au}, 100 \mathrm{GeV}$ operations from the RHIC IPMs and from the experimental ZDCs (red and black lines) from the FY11 Run (Fig. 6a, no channel-by-channel offset corrections and gain calibrations), from the FY14 Run (Fig. 6b, with channel-by-channel corrections and model beta functions at the IPMs), the same data from the FY14 Run (Fig. 6c, with measured beta functions at the IPMs only) and the same data from the FY14 Run (Fig. 6d, with measured beta functions at the IPMs and at STAR and PHENIX). The horizontal time scale is 3.5 hours in all cases.

\section{SUMMARY}

The accuracy of emittance measurements using the RHIC IPMs has been greatly improved by the following: (1) continual design enhancements over the years [4-8], (2) application of channel-by-channel offset corrections and gain calibrations in the beam profile measurements and (3) use of measured beta functions at the locations of the IPMs. The removal of systematic errors in the emittance measurements was confirmed by the convergence of all four planes of measurement (horizontal and vertical planes of both the Blue and Yellow beams) to a common value during beam operations with stochastic cooling (Fig. 5). Consistency with independent measurements (based on the ZDCs) at STAR and PHENIX was shown to be within 15\% (Fig. 6).

Future studies will involve continued efforts towards more precise measurements of the beta function during acceleration [15] and/or optics correction during acceleration [16] so that, together with measurements from the injectors, a better understanding of sources of emittance dilution between the AGS and RHIC and during acceleration in RHIC can be better characterized and localized and, eventually, corrected. 


\section{ACKNOWLEDGEMENTS}

The RHIC IPM design and evolution thereof has been led by R. Connolly. The beautiful 2008 design [8] had fabrication support by J. Fite, S. Jao, D. Kipp, T. Russo and C. Trabocchi and support from Vacuum, Controls and Instrumentation for installation and integration. S. Tepikian is the author of the fitting algorithm, which has been validated as robust and accurate, and who over the course of these studies provided continuous enhancements to the online data processing application. T. Summers championed implementation of the online calibration measurements. C. Liu's development and automation of the beta function interpolation routine [3] has been invaluable.

\section{REFERENCES}

[1] V. Ptitsyn, “Emittance” http://www.c-ad.bnl.gov/RHIC/retreat2006/presentations/PDF/emittance.pdf (with contributions by K.A. Drees, RHIC Retreat, Port Jefferson, NY (July, 2006).

[2] C. Adler et al, "The RHIC Zero Degree Calorimeters", Nucl. Instr. and Meth. in Phys. Res. A 470, pp. 488-499 (2001).

[3] C. Liu et al, "Global Optics Correction in RHIC Based on Turn-by-Turn Data from ARTUS Tune Meter", NAPAC13, Pasadena, CA, Sept 2013, TUPBA06, p.532 (2013).

[4] R. Connolly et al, “A Prototype Ionization Profile Monitor for RHIC”, PAC97, Vancouver, B.C., Canada, May 1997, p. 2152 (1997).

[5] R. Connolly et al, “The RHIC Ionization Profile Monitor”, PAC99, New York, NY, Mar 1999, p. 2144 (1999).

[6] R. Connolly et al, "Beam Profile Measurements and Transverse Phase-Space Reconstruction on the Relativistic Heavy-Ion Collider”, Nucl. Instr. and Meth. in Phys. Res. A 443, pp. 215-222 (2000).

[7] R. Connolly et al, "Residual-Gas Ionization Profile Monitors in RHIC”, PAC05, Knoxville, TN, May 2005, p. 230 (2005).

[8] R. Connolly et al, "Residual-Gas Ionization Beam Profile Monitors in RHIC”, BIW10, Santa Fe, NM, May 2010, TUPSM010, p. 116 (2010).

[9] G. Marr et al, "RHIC Performance for FY2011 Au+Au Heavy Ion Run”, Proc. of IPAC2011, San Sebastian, Spain, Sept 2011, TUPZ038, p. 1894 (2011).

[10] R. Connolly, M. Minty and S. Tepikian, "Calibration Scans on IPMs", APEX Weekly Meeting, http://www.cadops.bnl.gov/Instrumentation/InstWiki/index.php/File:APEX_IPM_presentation.pdf (2011).

[11] M. Blaskiewicz, J.M. Brennan and F. Severino, "Operational Stochastic Cooling in the Relativistic Heavy-Ion Collider”, Phys. Rev. Lett. 100, 174802 (2008).

[12] M. Blaskiewicz, J.M. Brennan and K. Mernick, “Three-Dimensional Stochastic Cooling in the Relativistic Heavy Ion Collider”, Phys. Rev. Lett. 105, 094801 (2010).

[13] W. Fischer, private communication.

[14] C. Dawson, private communication.

[15] M. Minty et al, "Measurement of Beam Optics During Acceleration in the Relativistic Heavy Ion Collider", NAPAC13, Pasadena, CA, TUPBA08, p. 538 (2013).

[16] C. Liu, A. Marusic, M. Minty, "Implementation of Optics Correction on the Ramp in RHIC", NA-PAC13, Pasadena, CA, TUPBA05, p. 529 (2013).

[17] A.W. Chao and M. Tigner, "Handbook of Accelerator Physics and Engineering”, World Scientific, p. 248 (1999).

[18] A.W. Chao and M. Tigner, ibid, p. 134. 


\section{APPENDIX A: TERMS CONTRIBUTING TO THE RMS BEAM SIZE}

The emittance $\varepsilon$ is given by the ratio of the square of rms beam size $\sigma_{\beta}$ to the beta function $\beta$ at the location of the IPM; i.e. $\varepsilon=\sigma_{\beta}{ }^{2} / \beta$ with $\sigma_{\mathrm{m}}{ }^{2}=\sigma^{2}+\sigma_{\eta}{ }^{2}+\sigma_{\mathrm{r}}{ }^{2}$, where $\sigma_{\mathrm{m}}$ is the measured rms beam size, $\sigma_{\eta}$ is the dispersive contribution to the beam size $\left(\sigma_{\eta}=\eta \delta\right.$, where $\eta$ is the dispersion function and $\delta$ is the beam energy spread) and $\sigma_{\mathrm{r}}$ is the measurement resolution. An example of the relative contributions to the beam sizes ${ }^{6}$, for which the emittances were displayed in Fig. 5 , is given in Table 3.

\begin{tabular}{|l|c|c|c|c|c|c|}
\hline & $\sigma_{\mathrm{m}}(\mathrm{mm})$ & $\eta(\mathrm{m})$ & $\delta$ & $\sigma_{\eta}(\mathrm{mm})$ & $\sigma_{\mathrm{r}}(\mathrm{mm})$ & $\sigma_{\beta}(\mathrm{mm})$ \\
\hline Blue Horizontal & 1.186 & 0.204 & $6.9 \mathrm{E}-4$ & 0.140 & 0.33 & 1.130 \\
\hline Blue Vertical & 0.799 & 0 & $6.9 \mathrm{E}-4$ & 0 & 0.33 & 0.727 \\
\hline Yellow Horizontal & 1.008 & 0.234 & $6.9 \mathrm{E}-4$ & 0.161 & 0.33 & 0.938 \\
\hline Yellow Vertical & 0.944 & 0 & $6.9 \mathrm{E}-4$ & 0 & 0.33 & 0.884 \\
\hline
\end{tabular}

Table 3: Terms contributing to the rms beam size $\sigma_{\beta}$.

The emittance, $\varepsilon$, displayed in Fig. 5 is the normalized, 95\% emittance, the latter defined as the phase space area containing $95 \%$ of a Gaussian beam; i.e.

$$
\varepsilon=6 \gamma \varepsilon_{\mathrm{rms}}=6 \gamma\left(\pi \sigma_{\beta}{ }^{2} / \beta\right)
$$

with $\gamma$ the Lorentz factor and $\beta$ the beta function at the location of the measurement. In Fig. 6, the emittance displayed omits the factor 6 and was referred to there as the rms emittance, $\varepsilon_{\text {rms. }}$.

\footnotetext{
${ }^{6}$ Data from 06/08/14, 18:31, fill 18403.
} 


\section{APPENDIX B: EMITTANCE FROM ZDCS CORRECTED BY MEASURED BETA FUNCTIONS}

The luminosity L is given [17] by

$$
\mathrm{L}=\mathrm{L}_{0} \mathrm{R}
$$

where $\mathrm{L}_{0}$ is the luminosity for infinitesimally short bunches and $\mathrm{R}$ accounts for finite bunch length (hourglass effect). In the limit that the horizontal and vertical beam sizes of the two colliding beams are equal at the interaction point, the luminosity for short bunches $\left(\sigma_{\mathrm{z}}<<\beta\right)$ with $\sigma_{\mathrm{z}}$ the bunch length and $\beta$ the beta function is [17]

$$
\mathrm{L}_{0}=\mathrm{N}_{1} \mathrm{~N}_{2} \mathrm{f}_{\mathrm{C}} / 4 \pi \sigma_{\mathrm{x}}^{*} \sigma_{\mathrm{y}}^{*}
$$

where $N_{1}$ and $N_{2}$ are the number of particles per bunch in beam 1 colliding with beam $2, f_{c}$ is the collision frequency and $\sigma_{\mathrm{x}}{ }^{*}$ and $\sigma_{\mathrm{y}}{ }^{*}$ are the horizontal and vertical beam sizes at the interaction point. With $\sigma^{2}=\varepsilon_{\mathrm{SA}}<\beta>$, where $\varepsilon_{\mathrm{SA}}$ is the emittance from StoreAnalysis [13], $\sigma=\sigma_{\mathrm{x}}{ }^{*}={\sigma_{\mathrm{y}}}^{*}$ and $\langle\beta\rangle=0.72 \mathrm{~m}$ the beta function averaged over all 4 planes (for the FY14 RHIC Run the beta functions in all planes were by design identical),

$$
\varepsilon_{\mathrm{SA}}=\mathrm{N}_{1} \mathrm{~N}_{2} \mathrm{f}_{\mathrm{C}} / 4 \pi<\beta>\mathrm{L}_{0}
$$

For the case of equal emittances but unequal beta functions at the IP (as the case with stochastic cooling [11, 12] as used during the FY14 RHIC Run),

$$
\mathrm{L}_{0}=\mathrm{N}_{1} \mathrm{~N}_{2} \mathrm{f}_{\mathrm{c}} / 2 \pi\left[\operatorname{sqrt}\left(\sigma_{1 \mathrm{x}}{ }^{2}+\sigma_{2 \mathrm{x}}{ }^{2}\right) \operatorname{sqrt}\left({\sigma_{1 \mathrm{y}}}^{2}+\sigma_{2 \mathrm{y}}{ }^{2}\right)\right]
$$

where $\sigma_{1 \mathrm{x}}$ and $\sigma_{2 \mathrm{x}}$ are the horizontal beam sizes at the interaction point of beam 1 and beam 2 and $\sigma_{1 \mathrm{y}}$ and $\sigma_{2 \mathrm{y}}$ are the vertical beam sizes at the interaction point of beam 1 and beam 2. Combining [B.3] and [B.4],

$$
\varepsilon_{\mathrm{SA}}=(1 / 2<\beta>)\left[\operatorname{sqrt}\left(\sigma_{1 \mathrm{x}}{ }^{2}+\sigma_{2 \mathrm{x}}{ }^{2}\right) \operatorname{sqrt}\left({\sigma_{1 \mathrm{y}}}^{2}+\sigma_{2 \mathrm{y}}{ }^{2}\right)\right]
$$

With equal emittances but unequal beta functions in all planes, the individual beam sizes at the interaction point are given by

$$
\sigma_{1 \mathrm{x}}^{2}=\varepsilon \beta_{1 \mathrm{x}}, \sigma_{2 \mathrm{x}}{ }^{2}=\varepsilon \beta_{2 \mathrm{x}}, \sigma_{1 \mathrm{y}}{ }^{2}=\varepsilon \beta_{1 \mathrm{y}} \text { and } \sigma_{2 \mathrm{y}}{ }^{2}=\varepsilon \beta_{2 \mathrm{y}} \text {. }
$$

Substituting into Eq. [B.5] gives

$$
\varepsilon_{\mathrm{SA}}=(\varepsilon / 2<\beta>)\left[\operatorname{sqrt}\left(\beta_{1 \mathrm{x}}{ }^{2}+\beta_{2 \mathrm{x}}{ }^{2}\right) \operatorname{sqrt}\left({\beta_{1 \mathrm{y}}}^{2}+\beta_{2 \mathrm{y}}{ }^{2}\right)\right]
$$

or

$$
\varepsilon / \varepsilon_{\mathrm{SA}}=2<\beta>/ \operatorname{sqrt}\left(\left(\beta_{1 \mathrm{x}} /<\beta>\right)^{2}+\left(\beta_{2 \mathrm{x}} /<\beta>\right)^{2}\right) \operatorname{sqrt}\left(\left(\beta_{1 \mathrm{y}} /<\beta>\right)^{2}+\left(\beta_{2 \mathrm{y}} /<\beta>\right)^{2}\right)
$$

From the beam optics measurements (reference footnote 1), and beam 1 representing the Blue Beam and beam 2 the Yellow Beam, the beta functions measured ${ }^{7}$ were

$$
\begin{gathered}
\beta_{1 \mathrm{x}}=0.830 \mathrm{~m}, \beta_{2 \mathrm{x}}=0.675 \mathrm{~m}, \beta_{1 \mathrm{y}}=0.670 \mathrm{~m} \text { and } \beta_{2 \mathrm{y}}=0.910 \mathrm{~m} \text { (at STAR) } \\
\beta_{1 \mathrm{x}}=0.645 \mathrm{~m}, \beta_{2 \mathrm{x}}=0.835 \mathrm{~m}, \beta_{1 \mathrm{y}}=0.745 \mathrm{~m} \text { and } \beta_{2 y}=0.855 \mathrm{~m} \text { (at PHENIX). }
\end{gathered}
$$

The corrections to the online emittance derived from the ZDCs is

$$
\begin{aligned}
& \varepsilon / \varepsilon_{\mathrm{SA}}=0.92(\mathrm{STAR}) \\
& \varepsilon / \varepsilon_{\mathrm{SA}}=0.87(\mathrm{PHENIX}) .
\end{aligned}
$$

\footnotetext{
${ }^{7}$ The beta functions were measured with non-colliding beams however the betatron tunes were $\sim 0.22$; that is, dynamic beta effects [18] may be neglected.
} 
The geometrical reduction in luminosity due to finite bunch length for Gaussian beams is given [17] by an integral (limits: - infinity to + infinity)

$$
\left.\mathrm{R}=(1 / \mathrm{sqrt} \pi) \text { integral exp(-t } \mathrm{t}^{2}\right) \mathrm{dt} / \operatorname{sqrt}\left[\left(1+\mathrm{t}^{2} / \mathrm{t}_{\mathrm{x}}^{2}\right)\left(1+\mathrm{t}^{2} / \mathrm{t}_{\mathrm{y}}^{2}\right)\right] \text {, }
$$

where

$$
\mathrm{t}_{\mathrm{x}}^{2}=2\left(\sigma_{\mathrm{x} 1}{ }^{2}+\sigma_{\mathrm{x} 2}{ }^{2}\right) /\left[\left(\sigma_{\mathrm{z} 1}+\sigma_{\mathrm{z} 2}\right)\left(\sigma_{\mathrm{x} 1}{ }^{2} / \beta_{\mathrm{x} 1}{ }^{2}+\sigma_{\mathrm{x} 2}{ }^{2} / \beta_{\mathrm{x} 2}{ }^{2}\right)\right]
$$

and similarly for $\mathrm{t}_{\mathrm{y}}{ }^{2}$. Assuming equal bunch lengths for both beams $\left(\sigma_{\mathrm{z}}=\sigma_{\mathrm{z} 1}=\sigma_{\mathrm{z} 2}\right)$ and using [B.6] for stochastically cooled beams, $\mathrm{t}_{\mathrm{x}}^{2}=\beta_{\mathrm{x} 1} \beta_{\mathrm{x} 2} / \sigma_{\mathrm{z}}$ and $\mathrm{t}_{\mathrm{y}}{ }^{2}=\beta_{\mathrm{y} 1} \beta_{\mathrm{y} 2} / \sigma_{\mathrm{z}}$ yielding

$$
\mathrm{R}=(1 / \text { sqrt } \pi) \text { integral } \exp \left(-\mathrm{t}^{2}\right) \mathrm{dt} / \operatorname{sqrt}\left[\left(1+\left\{\sigma_{\mathrm{z}} / \beta_{\mathrm{x} 1} \beta_{\mathrm{x} 2}\right\} \mathrm{t}^{2}\right)\left(1+\left\{\sigma_{\mathrm{z}} / \beta_{\mathrm{y} 1} \beta_{\mathrm{y} 2}\right\} \mathrm{t}^{2}\right)\right]
$$

Taking a representative measure (roughly midway in Fig. 6) for which the slowly varying bunch length was measured as $\sigma_{\mathrm{z}}=2.45 \mathrm{~ns}$ (FWHM), numerical integration of [B.13] yields $\mathrm{R}=0.411$ with model beta functions equal to $0.72 \mathrm{~m}$ in all 4 planes and, using the measured beta functions from Eq. [B.9], $\mathrm{R}=0.418$ (STAR) and $\mathrm{R}=0.418$ (PHENIX). The change in hourglass correction obtained using measured beta functions is therefore small: with the emittance $\varepsilon$ inversely proportional to $\mathrm{L}_{0}$ and $\mathrm{L}_{0}=\mathrm{L} / \mathrm{R}$ (from [B.1]), the emittance is proportional to $\mathrm{R}$. Therefore, the change in emittance due to use of the measured beta functions is $\mathrm{d} \varepsilon / \varepsilon=\mathrm{dR} / \mathrm{R}=0.418 / 0.411$ or $\mathrm{d} \varepsilon=1.017 \varepsilon$ (an increase by $1.7 \%$ ) at both STAR and PHENIX. 